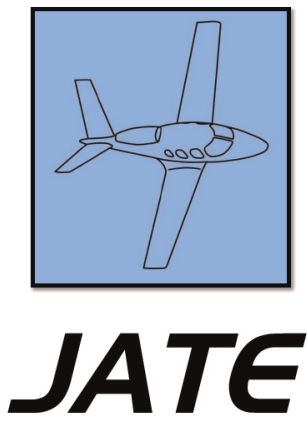

Journal of Aviation Technology and Engineering 1:2 (2012) 90-96

DOI: $10.5703 / 1288284314662$

\title{
Contributing Factors in Piper PA28 and Cirrus SR20 Aircraft Accidents
}

\author{
Andrew Franza and Richard Fanjoy
}

Purdue University

\begin{abstract}
A large Midwestern university has recently acquired Cirrus SR20 aircraft to replace an aging Piper PA28-161 fleet. The upgrade to new and more advanced Cirrus aircraft has led to major changes in the flight program curriculum. New training techniques, guidelines, and operating procedures have been introduced to facilitate a successful transition. This study compared contributing factors to fatal accidents in SR20 and PA28-161 aircraft to determine if there are new risks associated with the subject fleet change. Using National Transportation Safety Board data, this study examined probable cause and other pertinent data from fatal accidents over the past ten years. The data was statistically analyzed to determine if there is any correlation between contributing factors for the two aircraft types. This analysis will help university leaders identify new risks facing participants in the flight program. Recommendations based on the findings are offered with the intent of reducing the level of new risk associated with the fleet replacement.
\end{abstract}

Keywords: $\quad$ aircraft accidents, contributing factors, SR20, PA28

\section{Introduction}

Within the aviation industry, automated systems and digital avionics continue to replace more conventional, analog instrument suites. In July 2010, a major Midwestern university replaced its Piper PA28-161 fleet with Cirrus SR20 aircraft, introducing modern aviation technology into the flight program (Schenke, 2010). The introduction of this technically advanced aircraft (TAA) has brought about many changes to the university's flight program. This fleet replacement has

\section{About the Authors}

Andrew J. Franza is a graduate student at Purdue University in aviation and aerospace management. He holds an FAA Commercial Pilots Certificate with instrument and multi-engine ratings. His areas of research interest include advanced flight simulation and aviation business optimization.

Richard O. Fanjoy is the associate head of graduate education and research, Department of Aviation Technology, at Purdue University. Dr. Fanjoy's research agenda includes aviation human factors, cognitive models, and advanced flight instrumentation. He was a collaborator for the NASA project that evaluated employment of future heavy transport aircraft in the NextGen environment. A retired Air Force Command Pilot with extensive international experience, he has served as a heavy jet aircraft flight instructor, check airman, and aircraft accident investigator/board president. 
resulted in differences in training techniques, program rules, risk mitigation, and general operations. University faculty, staff, and students continue to adapt to the new aircraft and become more proficient each semester.

The Cirrus SR20 is representative of technological advances that have taken place in general aviation aircraft. While both the SR20 and PA28-161 are four seat, single engine aircraft, significant differences exist in the type of instrumentation each has. The SR20 has digital avionics, commonly called a glass cockpit, while the PA28-161 has analog instrumentation. The SR20 is classified as a TAA, in part, due to its advanced Garmin G1000 avionics suite and automated systems. Along with these differences, the SR20 has the added safety feature of a ballistic parachute designed to allow the aircraft to descend safely in the event of a loss of control or catastrophic airframe failure (Cirrus Aircraft, 2011). Even though both the PA28-161 and SR20 are considered by the Federal Aviation Administration (FAA) to be in the same category and class, enough differences exist between the two to warrant an examination of risk associated with operating the SR20 aircraft's glass cockpit and automation.

The purpose of this study was to determine if there are differences in the most frequent contributing factors leading to fatal accidents in PA28-161 and SR20 aircraft operated in the United States over the past 10 years. The findings of this study will enable flight training providers to make safety-related decisions based on a comparison of the previous and new aircraft fleet. It is hypothesized that there is a greater frequency of accidents as a result of pilot error in the SR20, when compared to the PA28-161, due to its advanced avionics suite.

\section{Accident Comparison: Glass Cockpit-Equipped Aircraft vs. Conventional Aircraft}

The target university's flight department, until recently, has provided flight training in aircraft with conventional analog instrumentation and very little automation. The flight training department's operating procedures, flight instruction techniques, and safety considerations reflect experience gained from operating these types of aircraft for many years. The introduction of the Cirrus SR20, which employs a digital avionics suite, commonly called a glass cockpit, and high levels of automation, necessitates a thorough review of literature and risk analysis to determine what changes must be made to the current flight curriculum. The Federal Aviation Administration (FAA), National Transportation Safety Board (NTSB), aircraft manufacturers, and academics have examined the safety impact of increased automation and glass cockpits. Findings from these investigations suggest that while glass cockpits and automation may be beneficial to pilots and reduce workload, they can also increase the chance of accident or incident if the pilot is not proficient with the systems (NTSB, 2010; Baxter, Besnard, \& Riley, 2007). To reduce the risk of accident in these more advanced aircraft, pilots must have a firm understanding of how the avionics and automation work.

Transport category aircraft have been equipped with digital instrumentation for many years but electronic displays have become standard for general aviation aircraft only in the past decade. The now common use of glass cockpits in light aircraft prompted the NTSB to examine the associated safety benefits and risks. In 2010, the NTSB completed a study focusing on how the transition to glass cockpits has affected the safety of light aircraft. The NTSB relied on manufacturer records, aircraft investigation information, and general aviation activity to compile a list of findings (NTSB, 2010). One of the major conclusions of the study was that aircraft accident and activity data showed a decrease in total accident rates but an increase in fatal accident rates for glass cockpit-equipped aircraft compared to conventionally-equipped aircraft (NTSB, 2010). The report suggests that the introduction of glass cockpits has not resulted in a measurable improvement in safety when compared to similar aircraft with analog instruments. The study also revealed that accidents involving aircraft equipped with glass cockpits tend to be associated with personal/business flights, longer flights, instrument flight plans, and single pilot operations, while accidents involving conventionally-equipped aircraft were more likely to be associated with instructional flights, shorter flights, and two-pilot operations (NTSB, 2010).

A significant finding of the NTSB's safety report was that training in conventional cockpits does not fully prepare pilots to operate aircraft with glass cockpits. Furthermore, the NTSB states that the FAA does not assess a pilot's knowledge of glass cockpit instrumentation and has no equipment-specific training requirements. These findings have led the NTSB to conclude that pilots may be poorly trained or incompetent to fly aircraft with glass cockpits when transitioning from more conventional aircraft (NTSB, 2010).

The NTSB study identified two safety issues: "The need for pilots to have sufficient equipment-specific knowledge and proficiency to safely operate aircraft equipped with glass cockpit avionics; and the need to capture maintenance and operational information in order to assess the reliability of glass cockpit avionics in light aircraft" (NTSB, 2010). Six recommendations were made as a result of the NTSB safety study, including revisions to airman knowledge tests to incorporate questions on glass cockpits and suggestions on the implementation of glass cockpit training. The NTSB also called on manufacturers to improve or add sections on electronic flight displays to their approved aircraft flight manuals and pilot's operating handbooks (NTSB, 2010). With these changes, the NTSB has determined that significant improvements in safety and reductions in accidents associated with glass cockpit aircraft can be achieved. 
While the NTSB study provides valuable information related to the safety of glass cockpit-equipped aircraft, it is important to understand that the findings may not be applicable to a training environment like the subject university's flight department. David Ison (2010) suggests that the NTSB report compares "apples to oranges." Ison supports this claim by stating that "glass airplanes are generally flown farther than conventional aircraft, deal with different weather conditions, are typically flown by a different pilot cohort, and tend to be used less for instructional flights than round dial types." It is imperative that the differences in typical operations be taken into account when comparing accident data of glass cockpit equipped aircraft with conventional aircraft. The NTSB findings provide a good background of safety issues related to glass cockpit equipped aircraft, but readers must fully understand the difficulties in comparing the accidents of TAAs, like the SR20, to conventional aircraft such as the PA28-161.

\section{Cognitive Mismatch with Advanced Systems}

A study by Baxter, Besnard, and Riley (2007) focuses on the risks associated with glass cockpit-equipped aircraft and examines the "cognitive mismatch" of automated systems and glass cockpits with their human counterparts. Their analysis explains how a pilot's inability to understand how the system is working and predict what it will do next can lead to an aircraft accident. The main focus of this examination is errors in the way flight crews in transport category aircraft perceive information presented in a glass cockpit environment, but the findings can be applied to general aviation aircraft with digital displays as well. The report found that blame for misinterpretation of digital instrumentation is due in large part to differences in the way humans think and differences in the way systems present data. This disagreement between mind and machine is defined as a cognitive mismatch. Baxter, Besnard, and Riley (2007) identify two types of mismatch, real and perceived:

Real mismatch occurs when there is an actual discrepancy between the pilot's mental model and the way that the system operates. This state of affairs is normally indicative of a flaw in the pilot's mental mode. A perceived mismatch occurs when the pilots perceive that there is an actual discrepancy between their mental model and the way that the system operates, when in reality there is not. In other words, this is state of affairs represents a false alarm. (Baxter et al., 2007)

Their study concludes by explaining that cognitive mismatches with aircraft systems should be a major concern and pilots flying an aircraft with a glass cockpit should be aware of possible errors. There is no single solution to the problem, and the best defense against cognitive mismatch is a pilot's understanding of how a system works and how data is presented. While the focus of this research is on instrument and system misinterpretations in multi-crew, transport category aircraft, cognitive mismatch is also a concern for pilots of light glass cockpitequipped aircraft like the SR20. The study suggests that there could be a greater chance for cognitive mismatch when pilots fly multiple types of aircraft with different digital displays. Different digital avionics platforms may operate and display information incongruously, making it possible for pilots to misinterpret data. For example, there are operational differences in Avidyne and Garmin glass cockpit suites typically used in advanced light aircraft. Proficiency in their particular glass cockpit aircraft helps pilots defend against a cognitive mismatch (Baxter et al., 2007).

\section{Flight Training}

Since university flight departments are primarily responsible for comprehensive flight training, it is critical to develop an understanding of risks associated with operating glass cockpit equipped aircraft. Michael Friday studied the opinions of designated pilot examiners (DPEs) performing flight evaluations in glass cockpit equipped aircraft. Friday surveyed a sample of DPEs to determine if they "perceive a problem with the current private pilot certification process with respect to the operation of [advanced display technology (ADT)] that could have a negative impact on aircraft safety" (Friday, 2011). Friday's survey revealed that $69 \%$ of DPEs feel that ADT created additional risk-taking behavior by pilots, which negatively impacted safety. This finding included flying in "lower visibility, hazardous weather, lower altitudes, closer to terrain, or closer to other aircraft" (Friday, 2011). The interviewed DPEs felt that the increase in risk taking behavior was the result of complacency, reliance on the technology, and the failure of the pilot to maintain awareness of their surroundings.

A study by John Di Renzo Jr. examined transition training from technically advanced aircraft to regional airline training. An interesting finding from this study is that 18 to 21 year olds often lack the discipline required to master high levels of automation (Di Renzo, 2009). The majority of students enrolled in the subject university flight program fall into this category. This finding suggests that those flight students may face more than normal difficulty when learning to fly in glass cockpit-equipped aircraft. Failure to gain an adequate understanding of advanced aircraft systems, such as the SR20's G1000 avionics, can result in higher levels of risk, increasing the chances of accidents.

Flight instructors often promote experience and currency as the best defense against accidents in technically advanced aircraft (Wallace, 2010; Hopkins, 2007). Those instructors argue that being proficient with the operation of glass 
cockpits found in TAAs will increase pilot awareness of errors in instrument interpretation and improper data entry. Lapsed proficiency in the aircraft's automation can lead to being surprised by the automated system's operations and response to pilot input (Sarter \& Woods, 1997).

\section{Federal Aviation Administration Initiatives}

In 2002, Robert Wright, a manager in the General Aviation and Commercial Division at the FAA, authored a white paper discussing the future of general aviation flight operations. Wright noted that a plateau in aircraft accidents along with advances in the National Airspace System and avionics calls for increased attention from the FAA (Wright, 2002). Wright predicted that further development of cockpit systems and avionics would necessitate improved flight training standards (Wright, 2002).

To address some of the concerns raised in Wright's white paper, the FAA developed FAA/Industry Training Standards (FITS). FITS is designed to reduce the number of general aviation accidents through the use of proven concepts in system safety for training in TAA (Federal Aviation Administration, n.d.). FITS uses scenario-based training encompassing risk management, aeronautical decisionmaking, situational awareness, and single-pilot resource management (Federal Aviation Administration, n.d.). Pilot training and aircraft types have changed very little over the course of aviation. Significant changes to avionics and aircraft systems have only been developed in recent years. FITS aims to aid instructors in teaching their students advanced systems often found in TAAs. The program is designed to adapt to changes as technology develops and aircraft systems advance (Federal Aviation Administration, n.d.). Implementing FITS into the subject university's flight curriculum would be beneficial and reduce the risks associated with flying technically advanced aircraft.

\section{Methodology}

The National Transportation Safety Board's Aviation Database was used as a data source for this study. The query function of the database allows users to specify a wide range of criteria such as aircraft type, timeframe, injury severity, and report status. For this project, the following search criteria were used:

Event Start Date: 09/01/2001

Event End Date: 09/01/2011

Investigation Type: Accident

Injury Severity: Fatal

Aircraft Make: Cirrus and Piper

Aircraft Model: SR20 and PA28-161

Report Status: Probable Cause

The search criteria yielded the data necessary to complete this study. The NTSB identification number, accident date, time of day, aircraft model, pilot age, pilot rating, pilot medical class, pilot total time, pilot total time in aircraft type, aircraft Hobbs time, meteorological information, and probable cause including the contributing factor and actual cause were all recorded in a database. Information that was not available in an NTSB report was left blank in the database.

An affinity diagram was chosen as the best tool to categorize and sort the data. Affinity diagrams allow for the "organization of large volumes of information efficiently and [identification of] natural patterns or grouping in the information" (Evans \& Lindsay, 2011, p. 204). After data was collected, NVivo 9 analysis software was used to categorize and group the data. Tools in NVivo 9 were used to organize the data and identify commonalities in probable cause and contributing factors to accidents across airframes. The word frequency function was used to identify the most common phrases in the probable cause and contributing factors of accidents for SR20 and PA28-161 aircraft. The parameters were set to search for matches of all words including synonyms. By using NVivo 9 to assist in the creation of an affinity diagram, the probable cause buckets were identified as pilot failure, mechanical malfunction, and environmental conditions. The contributing factors buckets were identified as loss of directional control, midair collision, collision with terrain, mechanical failure, and deviation from instrument procedures.

Next, Pareto diagrams were used to graphically present the data. A Pareto diagram was chosen because it graphically depicts how often the buckets contribute to accidents and their relative frequency to other contributors (Evans \& Lindsay, 2011, p. 551). In total, four Pareto diagrams were created, each graphically presenting data for probable cause and contributing factors across airframes. It is important to note that often times many factors contribute to the same accident. To accurately represent all aspects of probable cause and contributing factors of an accident, some may be recorded twice under different criteria. A graphical representation will allow decision makers to visually identify areas that need the most attention to improve safety. The bars in the Pareto charts represent frequency in descending order while the red line represents the cumulative percentage.

Finally, bar charts were created to compare the relative frequency of probable cause and contributing factors between SR20 and PA28-161 aircraft. These charts allow flight department decision makers to pinpoint differences in risk between the two airframes. From this, a list of recommendations was compiled to help mitigate the risk and address new levels of risk associated with SR20 aircraft.

\section{Research Limitations}

This study is intended to help university decision makers identify differences in risks between SR20 and PA28-161 
aircraft. It is important to note that the subject university uses SR20 and PA28-161 aircraft in training environments. While there are some circumstances of training-related fatal accidents recorded in the NTSB database used for this study, the majority of the accident data is unrelated to training. It is more common for a TAA to be involved in an accident while being flown for business or personal use as opposed to training. The conclusions of this study are also based on a few assumptions. For the purposes of this study, it is assumed that all aircraft are maintained airworthy and all pilots are current as required by regulation. This study did not take aircraft age into account. Data was collected from accidents over the past ten years without regard to aircraft age. Mechanical malfunction rates related to probable cause may be slightly higher for PA28-161 aircraft because they are typically older aircraft when compared to the SR20. Pilot qualifications were not considered but were available in the database. The database often provided insufficient and incomplete data on airmen, bringing the usefulness and relevance of pilot qualifications into question for this study. Finally, number of accidents per flight hour for each aircraft was not taken into account. This study examined accidents over a ten-year period without regard to accidents per flight hour. At the time of this research, that data was unavailable and the researcher determined that the aforementioned methodology would be sufficient for the purposes of this study.

\section{Results}

The data was categorized and presented in Pareto charts. Figures 1 and 2 represent probable cause of fatal accidents over the past ten years in the United States in SR20 and PA28-161 aircraft respectively. Figure 3 is a comparison of the relative frequency of accident causes between SR20 and PA28-161 aircraft.

The most common probable cause of SR20 and PA28161 fatal accidents over the past ten years in the United States was the pilot's failure to safely operate an aircraft.

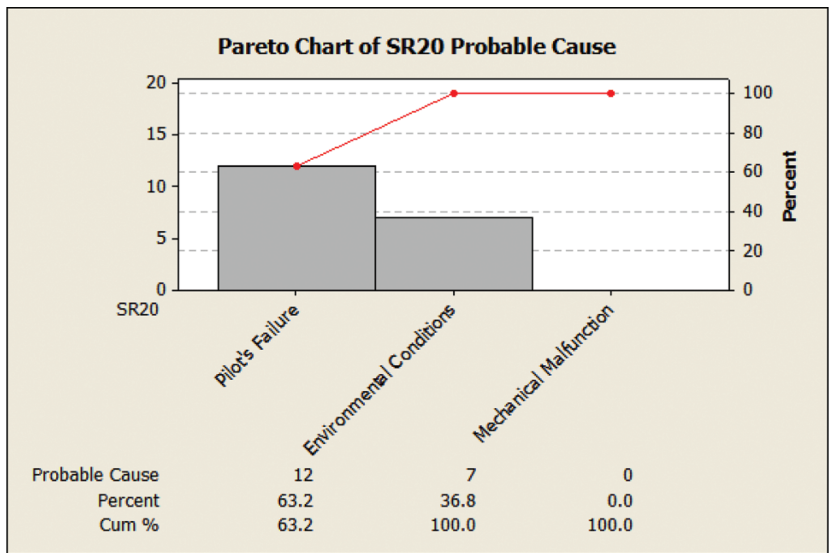

Figure 1. Pareto chart representing probable cause of fatal accidents involving SR20 aircraft in the United States over the past ten years.

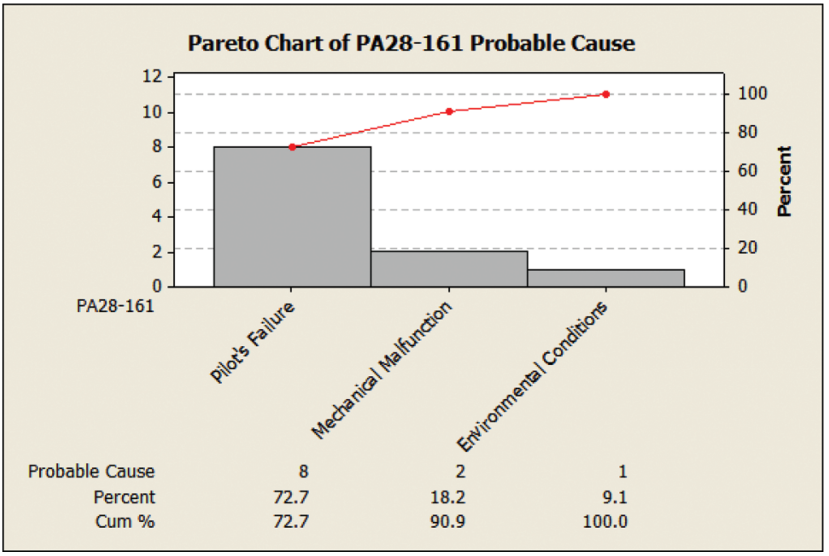

Figure 2. Pareto chart representing probable cause of fatal accidents involving PA28-161 aircraft in the United States over the past ten years.

This category represents any failure on the part of the pilot that led to an accident and can be equated to the term 'pilot error'. Environmental conditions were the second most common probable cause in fatal aircraft accidents in SR20 aircraft and the third most common probable cause in fatal accidents in PA28-161 aircraft. This category represents aircraft accidents as a result of any environmental conditions. Finally, mechanical malfunctions did not cause any accidents in SR20 aircraft but was the second leading probable cause of fatal accidents in PA28-161 aircraft. Comparison between the two aircraft types in Figure 3 indicates that pilot failure and mechanical function were more common in PA28-161 fatal accidents while environmental conditions were the probable cause in more SR20 fatal accidents.

Figures 4 and 5 represent the frequency of contributing factors to SR20 and PA28-161 fatal accidents in the United States over the past ten years, respectively. Figure 6 is a comparison of the contributing factors to accidents between SR20 and PA28-161 aircraft.

The analysis of contributing factors determined that loss of directional control was the primary contributing factor to fatal accidents in both aircraft types followed by collision with terrain. Deviation from instrument procedures was the

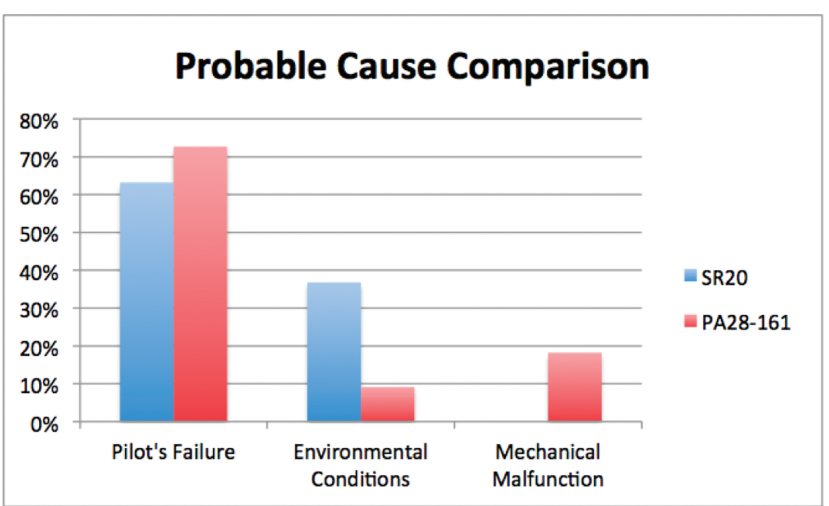

Figure 3. Comparison of the relative frequency of fatal accidents probable causes between SR20 and PA28-161 aircraft. 


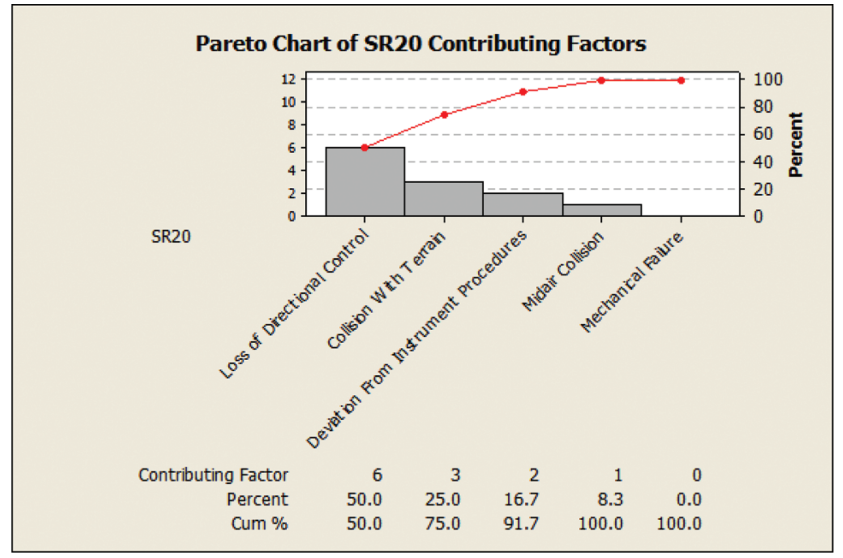

Figure 4. Pareto chart of representing contributing factors to fatal accidents involving SR20 aircraft in the United States over the past ten years.

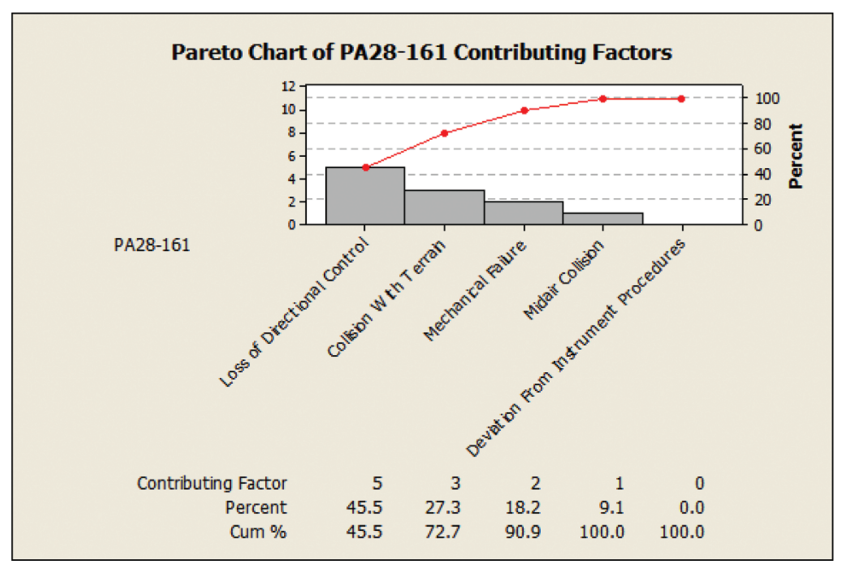

Figure 5. Pareto chart of representing contributing factors to fatal accidents involving SR20 aircraft in the United States over the past ten years.

third most common contributing factor to fatal accidents in SR20 aircraft but did not contribute to any PA28-161 accidents. Midair collision is fourth in the list of contributing factors to fatal accidents for both aircraft types. Finally, mechanical failures did not contribute to any SR20 aircraft accidents, but was the third contributing cause of fatal accidents in PA28-161 aircraft. Comparison

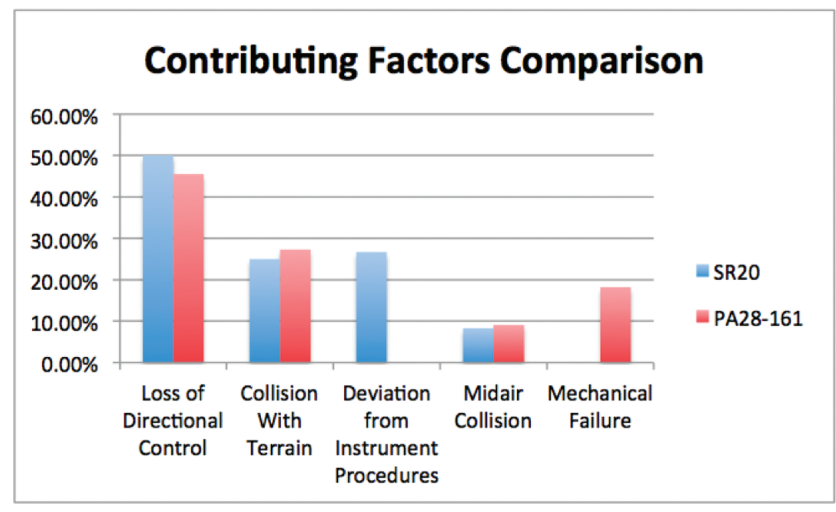

Figure 6. Comparison of the relative frequency of fatal accidents contributors between SR20 and PA28-161 aircraft. of the relative frequency of contributing factors to fatal accidents between both aircraft is graphed in Figure 6.

\section{Discussion}

From this analysis, it was determined that pilot error was more frequently the cause of fatal accidents involving PA28-161 aircraft over the past ten years when compared to SR20 aircraft. This result would suggest that the hypothesis of there being a greater percentage of accidents as a result of pilot error in the SR20, when compared to the PA28-161, due to its advanced avionics suite is false. This finding may be the result of multiple factors. One reason for this could be that more experienced pilots fly the SR20 aircraft when compared to PA28-161 aircraft. Only recently have flight schools and universities adopted SR20 aircraft and other TAAs as primary trainers. On the same note, aircraft such as PA28-161s are more frequently employed in all training environments. The relative experience of operators could account for the higher levels of pilot error with PA28-161s.

The study only recorded data on SR20 and PA28-161 aircraft, narrowing the focus to two specific airframes. Further study is recommended to determine if the findings occur across a population of all TAAs compared to all conventional aircraft. The findings of this study, however, suggest NTSB studies of TAA accident rates, relative to conventional light aircraft, are accurate. A more in-depth study should be completed at a later date, after TAAs in structured training environments are more commonly employed.

\section{Conclusion}

Technically advanced aircraft such as the Cirrus SR20 are the future of global aviation as automated systems and glass cockpit displays become more prevalent in light aircraft. As flight training fleets are converted to TAAs, it is important for students and instructors alike to understand the risks associated with these advanced aircraft. While the findings of this study may not identify specific risks associated with the SR20 aircraft, they suggest the most common causes of accidents when compared to the PA28161. As the subject university and other flight schools gain experience operating TAAs as primary trainers, specific safety measures and program modifications should be put in place to reduce risk and minimize the chances of incidents and accidents during flight operations.

Some preliminary recommendations have been compiled based on the findings of this study to ensure continued high levels of safety in flight departments that operate TAAs:

1. Implement TAA-specific accident and incident prevention measures.

2. Improve department-wide incident reporting to better track and assess risks associated with new TAA fleet.

3. Stress 'looking outside the aircraft' in visual meteorological conditions. 
4. Discuss levels of automation and techniques to mitigate risk during periods of high workloads.

\section{References}

Baxter, G., Besnard, D., \& Riley, D. (2007). Cognitive mismatches in the cockpit: Will they ever be a thing of the past? Applied Ergonomics. 38(4), 417-423, doi: 10.1016/j.apergo.2007.01.005.

Cirrus Aircraft. (2011). CAPS ${ }^{\mathrm{TM}}$ - live with it. Retrieved from http:// cirrusaircraft.com/innovation/?item $=$ parachute\#products

Di Renzo, Jr, J. C. (2009). The impact of transition training on adapting to technically advanced aircraft at regional airlines: Perceptions of pilot and instructor pilots. (Unpublished doctoral dissertation). Oklahoma State University, Stillwater.

Evans, J. R. \& Lindsay, W. M. (2011). Managing for quality and performance excellence. Mason, $\mathrm{OH}$ : South-Western Cengage Learning.

Federal Aviation Administration. (n.d.). Volume 1: FAA/Industry Training Standards. Retrieved from http://www.faa.gov/training_testing/ training/fits/training/flight_instructor/media/Volume1.pdf

Friday, M. A. (2011). A mixed methods study of designated pilot examiners experienced with advanced display technology and technology advanced aircraft. (Doctoral dissertation). Retrieved from ProQuest. (UMI 3459986).

Hopkins, J. (2007, August 9). Teaching glass. Flying, 134(8), 43.

Ison, D. (2010, August). Is glass safer? Plane \& Pilot, 46(7), 56.

National Transportation Safety Board. (2010). Introduction of glass cockpit avionics into light aircraft (NTSB Publication No. SS-01/10 PB2010-917001). Springfield, VA: National Technical Information Service.

National Transportation Safety Board. (2011). NTSB aviation database query. Retrieved from http://www.ntsb.gov/aviationquery/index.aspx

Sarter, N. B., \& Woods, D. D. (1997). Team play with a powerful and independent agent: Operational experiences and automation surprises on the Airbus A-320. Human Factors, 39(4), 553-569, doi: 10.1518/ 001872097778667997.

Schenke, J. (2010, July). Purdue takes delivery of new fleet of Cirrus aircraft [Press release]. Retrieved from http://www.purdue.edu/ newsroom/general/2010/100719M-BowenCirrus.html

Wallace, L. (2010, June 29). Flight school: Glass panel training. Flying. 137(6). 34.

Wright, R. A. (2002). Changes in general aviation flight operations and their impact on system safety and flight training. Retrieved from Federal Aviation Administration website: http://www.faa.gov/training testing/training/fits/guidance/media/whitepaper.doc 